\title{
EVALUATION OF A JET-PUMP FOR THE IMPROVEMENT OF OIL-WATER FLOW IN PIPELINE LOOPS USING CFD TOOLS
}

\author{
Miguel Asuaje $\mathrm{e}^{1,2^{*}}$, Jens Toteff $^{1}$, and Ricardo Noguera ${ }^{3}$ \\ ${ }^{1}$ Simón Bolívar University, USB, Energy Conversion Deparment, Valle de Sartenejas 89000, Caracas, Venezuela \\ ${ }^{2}$ Frontera Energy, Operations Technology Department, Calle 110 \# 9 - 25 Bogota, Colombia \\ ${ }^{3}$ École Nationale Supérieure d'Arts et Métiers, LIFSE - Arts et Métiers, Paris 75103, France
}

\begin{abstract}
Despite their low efficiency compared with centrifugal pumps, jet pumps are highly reliable, robust equipment with modest maintenance, ideal for many applications, mainly in the oil \& gas industry. Jet pumps are conventionally used to draw fluid from a storage tank in the petrochemical industry or as an artificial lift system to produce oil from a reservoir using energy from the primary fluid. The trunk lines in oil production systems can experience an unfavourable phenomenon due to the fluid's low velocities. In the case of transporting a heavy oil-water mixture with low flow velocities, it could promote oil and water stratification. Due to high viscosity, the stratified oil stick on the pipe,| causing a diameter reduction, resulting in a drop in fluids production and increased energy consumption. Given the virtue of jet pumps, this paper proposes using this equipment as an oil-water transfer pump as an alternative to expensive conventional multiphase pump systems. The jet pump will add fluid into the line, increase the fluid velocities, and promote the homogenous mixture of oil and water. Using ANSYS CFX, the effect of installing a jet pump in a conventional trunkline loop was analysed. Three jet pump configurations were simulated for different driving fluid pressures and compared against a traditional pipeline loop's performance. The first configuration shows a poor performance increasing only until $10 \%$ of handling fluids. Conversely, with the improved jet pump configurations rise of the fluid production by $30 \%$ has been obtained.
\end{abstract}

\section{Introduction}

Ejectors are devices with no moving parts using fluids under controlled conditions. With a high-pressure driving flow, they boost a low-pressure flow discharging at intermediate pressures. The operation principle converts the motive fluid's total pressure (primary fluid) into velocity through a nozzle. The high speed creates a low-pressure zone in the suction chamber causing secondary fluid to be pumped into the suction chamber. The two liquids are mixed by exchanging momentum in the ejector's neck. Total mixture flow goes into the diffuser, where hydraulic energy is recovered until an intermediate pressure. Figure 1 shows the parts of the ejector.

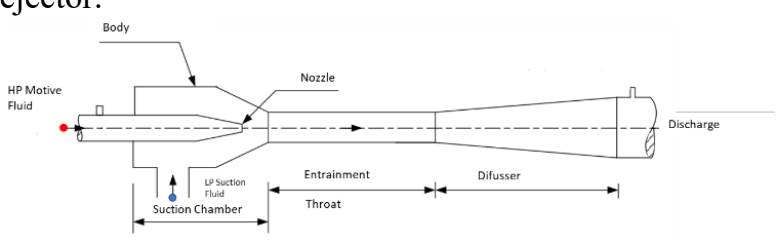

Fig. 1. Jet Pump diagram.

Due to the simplicity in its design, easy installation, high operational reliability, and handling of fluids of any kind, the ejectors have been of great interest for their industrial application like transport of solids, wastewater, booster pumping, pumping of abrasive and corrosive liquids, among others. Its performance is usually calculated under the following equations:

$$
\begin{gathered}
N=\frac{H_{d i s c}-H_{L P}}{H_{H P}-H_{\text {disc }}} \frac{\rho_{L P}}{\rho_{H P}} \\
M=\frac{Q_{H P}}{Q_{L P}} \\
\eta=M \cdot N
\end{gathered}
$$

The ejector theory was suggested by O'Brien in 1934 [1], who established the governing equations. Later, many researchers added corrective terms due to friction, such as Cunningham and River's [2]. The theoretical formulation assumes that the primary and secondary flows enter the neck with uniform velocity distributions. The mixed flow exits the neck and diffuser with a uniform velocity profile [2] [3]. Figure 2 graphically shows the uniform velocity profiles inside the Pump. Given the asymmetry of the equipment and the suction speed, this assumption may not be fulfilled [4].

\footnotetext{
* Corresponding author: $\underline{\text { asuajem } @ \text { usb.ve }}$
} 


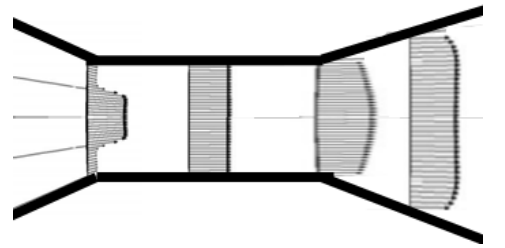

Fig. 2. Nozzel and Neck Ideal Velocity Profil.

\section{Analysis and Modelling}

\subsection{Case Study}

In the oil and gas industry, mature wells' global presence increases over the years. These reservoirs usually produce high water content and are primarily associated with heavy crude oils with low gas fractions [5]. Over the production life of the reservoir, the trend is to increase the water content. It is estimated worldwide that at least three water barrels on average are produced for every barrel of oil produced. On the other hand, reservoirs with nearby aquifers also tend to have high water content. For high water cut production wells, the ideal flow pattern for transporting to the treatment facilities is a homogeneous flow. Despite the high-water cut ( $>90 \%$ ), the gathering system could experience some transport problems due to premature and unwanted phase separation; very frequently, as a consequence of the length of the pipes, terrain topography, and oversizing of the diameters of the lines, under the premise that with a larger diameter more fluid is transported. This problem, which is a consequence of low speeds on the pipelines, could worsen when incorporating a loop or relief connection into the main production lines. These loops are typically constructed, as shown in Figure 3.

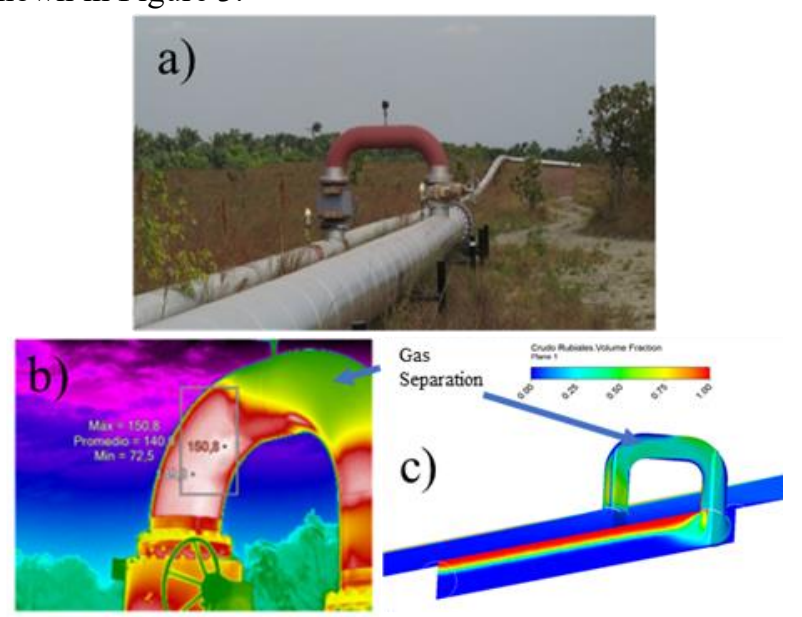

Fig. 3. a) Pipeline Loop in oil production Trunkline in Quifa Field, b) Loop Thermography, c) Oil Fraction by CFD [5]

A custom-designed ejector can operate as a booster pump assisted by a smaller single-phase pump providing a high-pressure motive fluid. Jet pump supply energy and flowrate to boost the total flow handling avoiding separation. Figure 4 shows a simplified scheme of the pipeline loop with and without a booster jet pump.
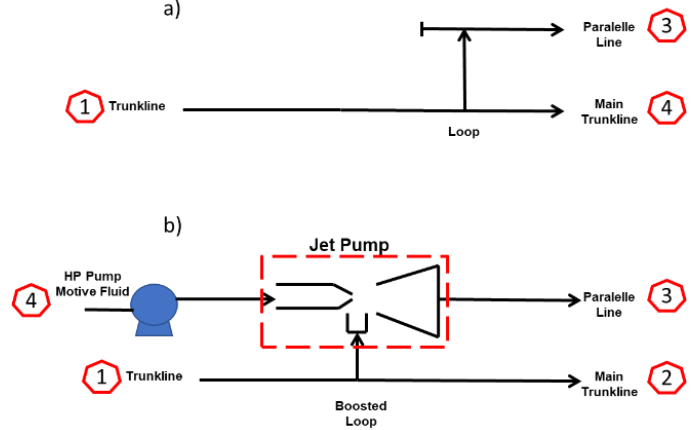

Fig. 4. Booster Jet Pump installed in a Trunkline loop scheme. a) without jet pump, b) with jet pump

\subsection{Jet Pump Design}

The ejector design and the selection of all geometrical parameters were carried out by an optimization process combined with CFD results described in previous works [6]. Figure 5 shows design parameters, CFD grid, and optimization results for the jet pump [6].
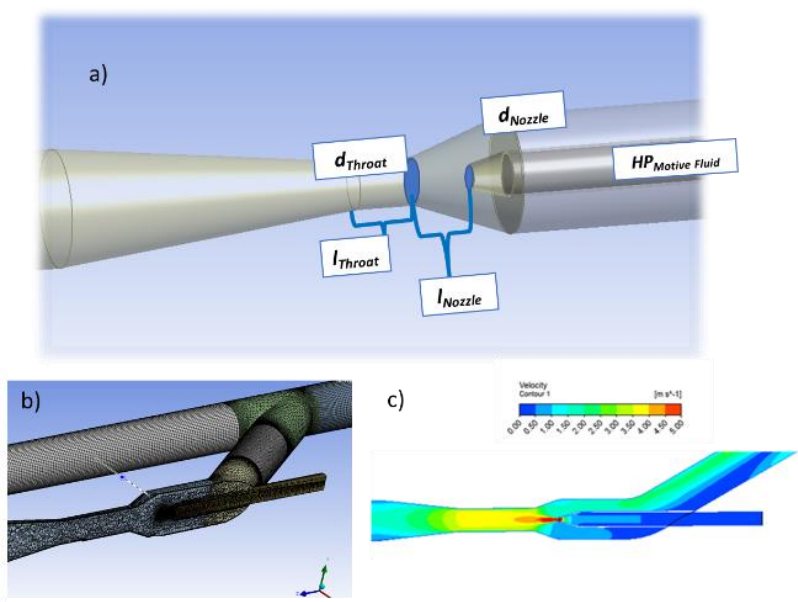

Fig. 5. Jet Pump Design: a) Geometrical Variables for Optimization Process, b) CFD Grid, c) Velocity Field inside de Pump

\subsection{Physical Domain}

Figure 6 shows part of the domain. The pipe nominal diameter of the principal and relief line is 24 ". The mainline's total length is $25 \mathrm{~m}$. The relief line is parallel connected by a pipe of $1.5 \mathrm{~m}$. The original loop (like Figure 3 ) is located $10 \mathrm{~m}$ from the discharge. The pipe directed towards the nozzle is 8 " in diameter, and the suction chamber, intake line, and discharge of the diffuser are 24 " to be coupled to the system without additional fittings.

Figure 6 shows the loop without a jet pump and the three configurations studied to install the ejector. The first configuration is called the standard $90^{\circ}$ mainline flow. Options 1 and 2 represent an improvement by deriving the flow from the main lines with a $30^{\circ}$ fitting. 


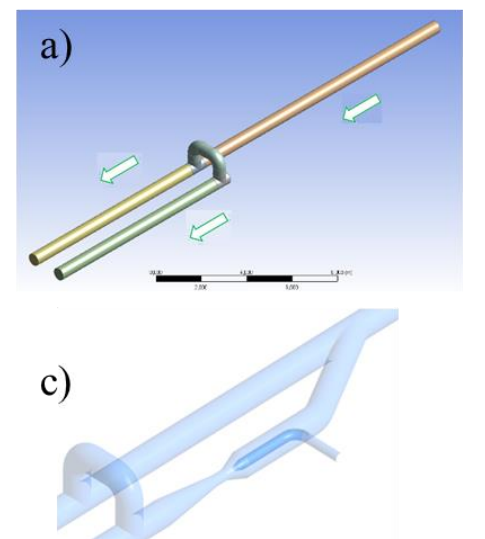

b)

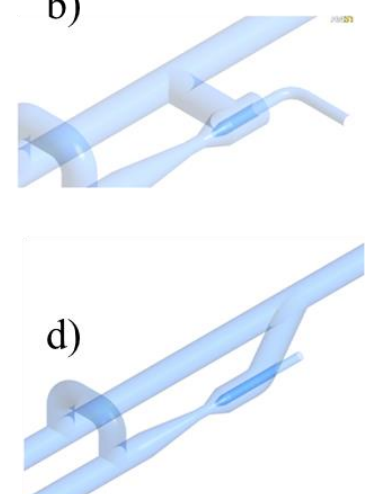

Fig. 6. Fluid physical domain a) Original Loop, b) Jet Pump Standard installation, c) Jet Pump option 1, d) Jet Pump option 2

\subsection{Grid}

An independent mesh study was carried out. Three grid sizes were used: The fine mesh was made with 2100.000 , the medium grid with 3800.000 , and the fine grid with 6400.000 . Simulations were performed over different inlet boundary conditions, obtaining associate errors of less than $1 \%$. More details are reported in Toteff, 2015 [7].

\subsection{Fluids Properties}

Water and oil properties are summarized in table 1. The input fluid is a homogeneous oil-water mixture, with a water cut of $96 \%$. The motive fluid is water.

Table 1. Fluid Properties @ $25^{\circ} \mathrm{C}$.

\begin{tabular}{|c|c|c|}
\hline $\begin{array}{c}\text { Property } \\
\text { /Fluid }\end{array}$ & Water & Oil \\
\hline $\begin{array}{c}\text { Density } \\
{[\mathrm{kg} / \mathrm{m} 3]}\end{array}$ & 997.00 & 974.78 \\
\hline $\begin{array}{c}\text { Viscosity } \\
{[\mathrm{cp}]}\end{array}$ & 1.00 & 277.45 \\
\hline
\end{tabular}

\subsection{Numerical Approach}

The computational calculations were performed using ANSYS-CFX codes, solving the Navier-Stokes average Reynolds equation. Simulations were carried out assuming steady-state, incompressible, isothermal fluids under gravitational effect. In an Euler-Euler approach, oil droplets dissolved in water were considered as a homogeneous mixture. As suggested by Aldas and Yapici [7], SST turbulence model is used for the continuous phase due to its ability to predict boundary layer behavior. The SST turbulence model is a combination of the $\kappa-\varepsilon$ model in the region outside the boundary layer and $\kappa-\omega$ in the inner zone of the boundary layer. A summary of numerical conditions is shown in table 2.
Table 2. Summary of simulation conditions.

\begin{tabular}{|c|c|}
\hline Parameter & Details \\
\hline Fluids & Water - Oil \\
\hline Wcut & $94 \%$ \\
\hline $\begin{array}{c}\text { Turbulence } \\
\text { model }\end{array}$ & SST \\
\hline Solving & Steady State \\
\hline $\begin{array}{c}\text { Advection } \\
\text { Scheme }\end{array}$ & 2nd Order \\
\hline Stop criteria & $\begin{array}{c}\text { For mass and } \\
\text { momentum: }\end{array}$ \\
\hline
\end{tabular}

The boundary conditions are presented in table 3. Low Total pressure and outlet pressures were kept constant. The high-Pressure motive fluid which represents, the energy supplied to the ejector, was varied.

Table 3. Boundary conditions.

\begin{tabular}{|c|c|}
\hline Parameter & Condition \\
\hline $\begin{array}{c}\text { 1 Main Pipeline Suction } \\
\text { Fluid Inlet LP }\end{array}$ & $\begin{array}{c}\text { Total Pressure } \\
{[100 \text { psia] }}\end{array}$ \\
\hline $\begin{array}{c}\text { 2 Main Pipeline Outlet } \\
\text { Flow }\end{array}$ & $\begin{array}{c}\text { Static Pressure } \\
{[98 \text { psia] }}\end{array}$ \\
\hline 3 Loop Outlet Flow & $\begin{array}{c}\text { Static Pressure } \\
{[98 \text { psia }]}\end{array}$ \\
\hline 4 Motive Fluid Inlet HP & $\begin{array}{c}\text { Total Pressure } \\
{[\text { Variable] }}\end{array}$ \\
\hline
\end{tabular}

\section{Results and discussion}

In production transport systems, parallel lines or loops are incorporated to increase production by reducing bottlenecks due to friction losses. A nondimensional inlet flowrate is defined to quantify the impact of installing the booster pumps. This is:

$$
Q_{1}^{*}=\frac{Q_{1 \text { Booster }}}{Q_{1 \text { Loop }}} 100 \%
$$




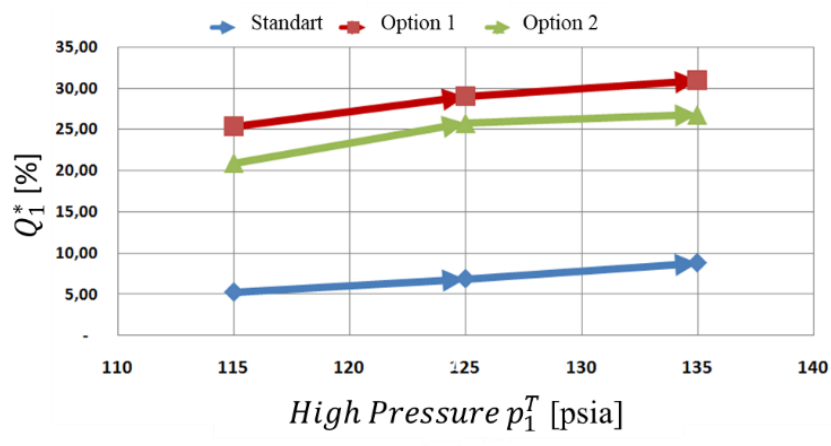

Fig. 7. Nondimensional Flow Rate in the loop using a Jet Pump

Figure 7 shows the percentage of increase of total flow rate by using a booster jet pump. Standard $90^{\circ}$ connection offers a poor performance compared against de option 1 and 2, increasing barely $10 \%$ for the motive fluid's same power condition. Using a $30^{\circ}$ fitting provides better performance for options 1 and 2 , increasing the total flow rate until $30 \%$ over the loop's reference flow. Between options 1 and 2, there is a constant difference of $5 \%$ aprox in the total flow rate. Poor behavior on standard jet pump connection can be explained by the diffuser's nonuniform velocity field, as shown in figure 8. Cunningham described this condition previously, even though the design criteria suppose a uniform inlet and outlet flow [4].

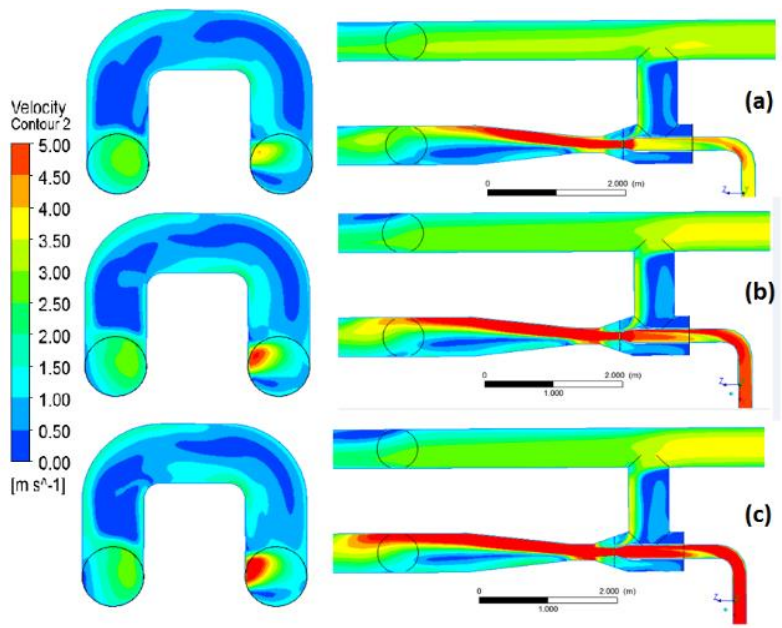

Fig. 8. Velocity fields for Jet Pump standard installation at different HP inlet conditions a) 115 psia, b) 125 psia, c) 135 psia

This exit recirculation results from a nonuniform velocity inlet into the suction chamber. Note that regardless of pressure, there is a strong recirculation at the low-pressure ejector intake. So, the suction area's effectiveness is significantly reduced, causing a flow restriction for all high-pressure conditions.

In this configuration, the low-pressure fluid flow has radial entry into the throat, which produces a deflection in the motive fluid line, causing prematurely fluid flow detachment in the diffuser.

On the other hand, proposed options 1 and 2, figures 9 and 10 respectively, seem to significantly reduce the effect of asymmetry and vortices at the inlet, improving the overall ejector performance.

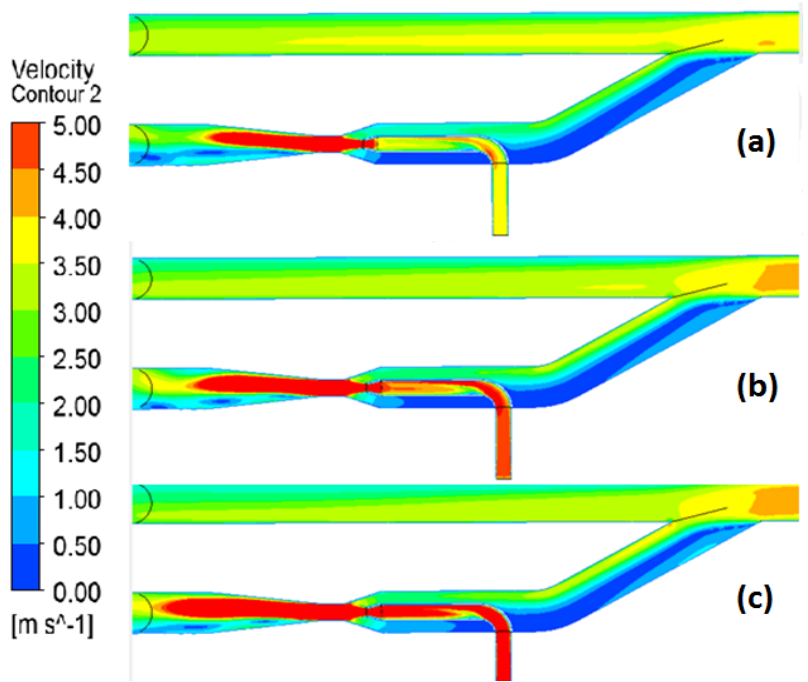

Fig. 9. Velocity fields for Jet Pump under option 1 installation at different HP inlet conditions a) 115 psia, b) $125 \mathrm{psia}, \mathrm{c}) 135 \mathrm{psia}$

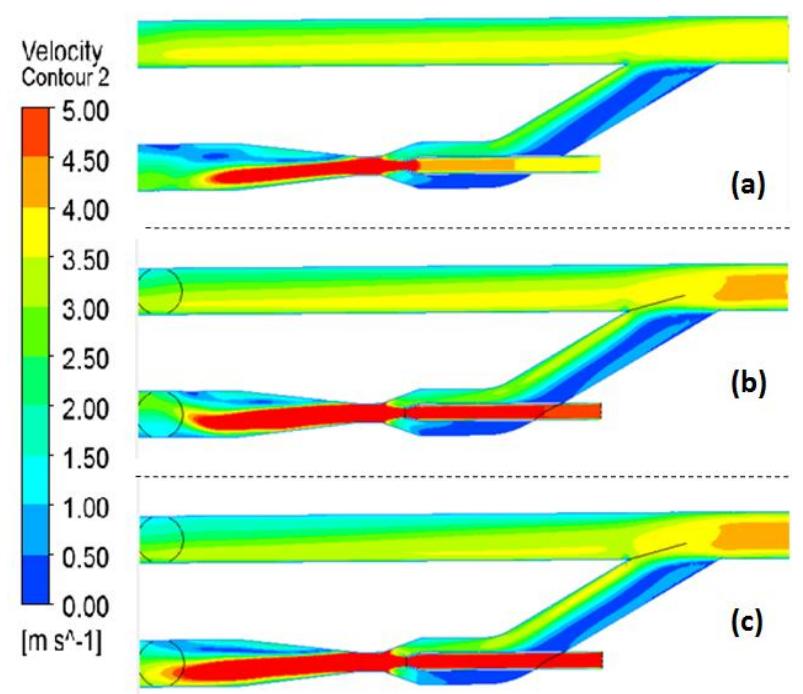

Fig. 10. Velocity fields for Jet Pump under option 2 installation at different HP inlet conditions a) 115 psia, b) 125 psia, c) 135 psia

This behavior is consistent with using a $30^{\circ}$ fitting which is recommended for a flow bypass. Nevertheless, low velocities at the low-pressure side and recirculation at the diffuser are still present, but

They do not block the total fluid passing through the Pump.

Other nondimensional flow rates have been defined to analyze the fluid distribution in the pipe

$$
\begin{aligned}
Q_{4}^{*} & =\frac{Q_{4}}{Q_{3}} 100 \% \\
Q_{3}^{*} & =\frac{Q_{3}}{Q_{1}} 100 \%
\end{aligned}
$$




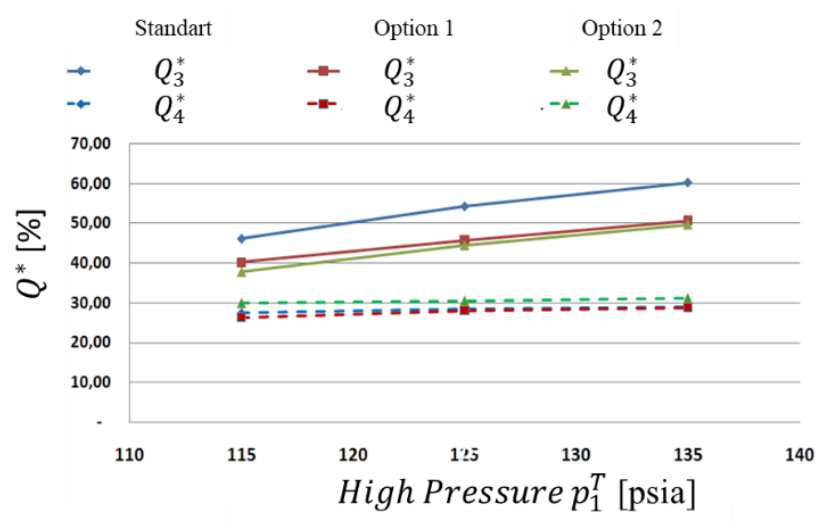

Fig. 11. Nondimensional flows distribution in trunk line loop with a Jet pump

Figure 11 shows that motive fluid relation with the outlet flow at the loop is constant for any high-pressure inlet condition. In other words, the fluid motive flowrate will be approximately $30 \%$ of the flow exing at the parallel line. Concerning the inlet flow, for option 1 and 2 , the parallel line will handle up to $50 \%$ of the total produced fluid.

To better illustrate, Figure 12 shows values expressed in Barrels per day for a high-pressure value of 125 psia. As the difference in both proposals' performance is not significant, option two should be selected for its simple construction and installation conditions.

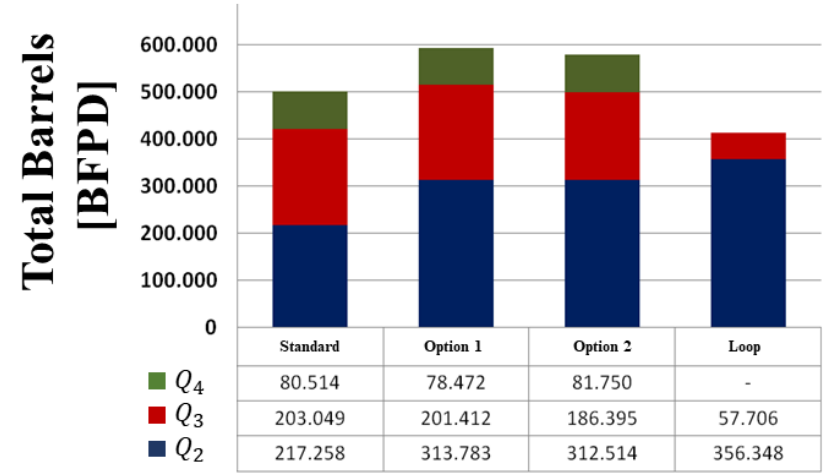

Fig. 12. Fluid distribution in the loop with and without jet pump at 125 psia

\section{Conclusions}

1. Performance analysis of a trunkline oil gathering system was carried employing CFD.

2. After study loop behavior without a jet pump, three configurations installing a jet pump were analyzed, the standard connection at $90^{\circ}$ and two connections at $30^{\circ}$.

3. Poor performance was obtained for standard jet pump connection, reaching a $10 \%$ of the extra flow barely.

4. Results show an improvement in total handled flowrate over $30 \%$ using either option one o option two.

5. Internal velocity fields presented reinforce these results and delight that the assumption of uniform flow through the body of the Pump could be wrong.
6. The flow rates ratio between the motive fluid line and the exit of the parallel line seems to be a constant value near of $30 \%$.

\section{Recommendations}

It is recommended to validate the performance of the jet pump for proposed options experimentally.

\section{Acknowledgments}

The authors gratefully acknowledge Frontera Energy for having supported this research.

\section{References}

1. J. Gosline, M. O'Brien, The Water Jet Pump. Univ. of California Pub. in Eng, 3 (1934)

2. R. G. Cunningham, W. River, Jet-pump theory and performance with fluid of high viscosity. ASME, 79 (1957)

3. I. Karassik, Pump Handbook. 2nd ed. New York: McGraw-Hill, (1986)

4. R. G. Cunningham, Liquid jet pump modeling: Efects of axial dimensions on theory experiment agreement. 2nd Symposium on Jet Pumps and Ejectors and Gas Lift techniques, March (1975).

5. M. Asuaje, N. Benitez, D. Quintero, M. Gaviria, E. Díaz, C. Chacón, "Improvements in the Dehydration Process of Heavy Crude Oil, Using CFD. Case Study Campo Quifa. Colombia" FEDSM 2018-83442. ASME 5th Joint USEuropean Fluids Engineering Division Summer Meeting, Montreal, Quebec, Canada (2018) https://doi.org/10.1115/FEDSM2018-83442

6. J. Toteff, M. Asuaje, "Design and Multiparameter Optimization of Jet-Pumps in a Pipeline Loops Using CFD Tools", FEDSM 2018-83440. ASME 5th Joint US-European Fluids Engineering Division Summer Meeting, Montreal, Quebec, Canada (2018) https://doi.org/10.1115/FEDSM2018-83440

7. J, Toteff, "Dimensionamiento y análisis de desempeño de eyectores en conexiones de líneas de alivio para el transporte de crudo" Universidad Simón Bolívar, Venezuela (2015)

8. Aldas, K., Yapici, R., "Investigation of effects of scale and surface roughness on efficiency of water jet pumps using CFD" Engineering Applications of Computational Fluid Mechanics, 14-25 (2014) 